\title{
Automated Contrast Improvement of X-Ray Image Using Otsu Threshold on Contrast-Limited Adaptive Histogram Equalization Algorithm
}

\author{
Aditya Akbar Riadi ${ }^{1}$, Esti Wijayanti ${ }^{2}$, Wibowo Harry Sugiharto ${ }^{3}$, \\ Anastasya Latubessy ${ }^{4}$, Alif Catur Murti ${ }^{5}$ \\ \{aditya.akbar@umk.ac.id ${ }^{1}$, esti.wijayanti@umk.ac.id², wibowo.harrys@umk.ac.id ${ }^{3}$ \} \\ Departement Informatics Engineering, Universitas Muria Kudus, Indonesia ${ }^{12345}$
}

\begin{abstract}
In the medical world, X-ray image quality is still a serious concern. The quality of X-ray image results is represented in the X-ray image contrast range. Correction of contrast ranges can be done computationally to improve X-ray image quality. From the problem then needed a method to improve result of X-ray image quality. In this research proposed OTSU thresholding modified into the Contrast-Limited Adaptive Histogram Equalization algorithm as a substitute for cliplimit value to improve the contrast quality of X-ray Image results which will then be measured with Mean Square Error to find out how much error value is generated.
\end{abstract}

Keywords: OTSU thresholding, contrast-limited adaptive histogram equalization, mean square error.

\section{Introduction}

The use of X-Ray images (called rontgen) in the medical world is very helpful for doctors and radiologists in diagnosing a disease to determine the type of treatment and subsequent treatment in patients, especially for patients fractures. Imaging using X-rays has been done to scan the various organs and tissues of the human body, among other fractures in bone, sinus examination, breast examination (mammography), examination of the heart, skull examination, spinal examination, urinary bladder kidney examination, examination of eye sockets, twelve finger intestine examination, gallbladder examination, and lower gastrointestinal tract examination [1]. This becomes something interesting because X-Ray image is obtained using the help of X-rays that can penetrate the skin and human flesh. It can not be used on a regular digital camera but uses a special tool [2]. The results of rontgen or X-ray images are either clear or appropriate to the desired ones, but of course many of the results of X-ray Image are inaccurate or unreadable by the viewer. For that required algorithm method that can improve the quality of X-ray Image results [3]. The results of rontgen or X-ray images that have not been done to improve the quality using algorithmic methods, usually influenced by several factors to be poured the author in the identification of the problem below.

There are some things from the X-ray Image or Radiography that we need to know and distinguish the clear and decisive form, detail or definition that shows the sharpness of the object seen until the smallest part of the object, the difference light and dark (white and black) contrast results X-ray and distortion or shape size changes on X-ray images.[4]

ICCSET 2018, October 25-26, Kudus, Indonesia

Copyright (C) 2018 EAI

DOI 10.4108/eai.24-10-2018.2280550 
Meanwhile, X-rays have an effect or a very harmful effect on our body that is when used in excess because it will be able to cause dangerous diseases, such as cancer. Therefore doctors do not advocate overuse of "rontgen photos". If there is an error in taking X-ray photographs by experts (doctors or radiologists) or X-Ray image results are less sharp results, in order to avoid irradiation of X-ray beams repeatedly then the results of X-rays taken earlier can be done some stage method that can improve the quality of X-ray image results.

From the general problem above, many researches are done to overcome the problem. This means that research on quality improvement issues for X-ray image result is still being done to find new methods that can solve the problem. In this research OTSU thresholding is used to fill its cliplimit value into the Contrast-Limited Adaptive Histogram Equalization algorithm which will then be measured by MSE to find out how much error value is generated.

\section{Research Method}

In this research there are three important steps that are used, the first is the image test that has been prepared with CLAHE algorithm, the second step of each image is calculated threshold value with OTSU, the third step of contrast automation process with the combination of CLAHE algorithm with OTSU threshold value.

\subsection{Contrast-Limited Adaptive Histogram Equalization}

Contrast Limited Adaptive Histogram Equalization (CLAHE) includes image repair techniques used to improve image contrast. CLAHE fix local contrast on image. CLAHE is a generalization of Adaptive Histogram Equalization (AHE). Unlike the equalization histogram that operates on the entire region of the image, CLAHE operates on a small region on a grayscale image called a tile. The contrast on each tile is fixed so that the histogram generated from that region roughly matches the specified histogram shape. The neighboring tiles are connected by using bilinear interpolation. This is done so that the result of tile merging looks smooth. Contrast, especially in homogeneous areas, may be limited to avoid any noise gain that may be present in the image.[5]

AHE (Adaptive Histogram Equalization) is a technique for improving image contrast by increasing the local contrast of the image. Local image is obtained by forming a symmetric grid in the image called region size [6]. Problems of increasing overly high contrast on AHE (Adaptive Histogram Equalization) that can be overcome by using CLAHE, which gives the limit value on the histogram. This boundary value is called the clip limit which represents the maximum height limit of a histogram. How to calculate CLAHE can be defined as follows:

$$
\beta=\frac{M}{N}\left(1+\frac{\alpha}{100}\left(s_{\max }-1\right)\right)
$$

The variable $M$ is the area size area, $N$ is the grayscale value (256), and $\alpha$ is the clip factor represents the addition of the limit of a histogram of values 0 to 100 .

The histogram above the clip limit is considered to be the excess of the pixels to be distributed to the surrounding area under the limit clip so that the histogram is evenly distributed. Illustration of the distribution of excess pixels in the above formula.

In CLAHE formula in MATLAB is adapthisteq, where Cliplimit CLAHE value must exist between 0 and 1 with normal value. for example cliplimit 0.5 means 127.5 for image uint 8 and 1.0 means is 255. Cliplimit value higher than lower cliplimit will spread further, therefore more contrast. 
CLAHE limits the amplification of clipping histograms at predefined values before calculating CDF (Cumulative Distribution Function). This limits the slope of the CDF (Cumulative Distribution Function) and the transformation function. Where the value of a truncated histogram, called cliplimit, depends on the normalization of the histogram and with adjacent regions. It is advantageous not to dispose part of the histogram over cliplimit but to distribute it equally among all the rest of the histogram.

The CLAHE parameter in matlab "NumTiles" determines the number of rectangular contextual regions (tiles) in which the adapthisteq divides the image. adapthisteq calculates the contrast changing functions for each region individually. The optimal number of tiles depends on the type of input image, and the best is determined through the experiment.

"Cliplimit" is a contrast factor that prevents over-saturation of images especially in homogeneous areas. This area is marked by a high peak in the histogram of a particular image tile because many pixels fall within the same gray level range. Without clip boundaries, adaptive equalization histogram techniques can produce results that, in some cases, are worse than the original image.[7]

"Distribution" defines the distribution that adapthisteq uses as a basis for creating contrast transformation functions. The distribution you choose should depend on the type of input image. For example, underwater imagery appears to be more natural when Rayleigh distribution is used.

\subsection{OTSU Thresholding}

The Otsu method was published by Nobuyuki Otsu in 1979 in his A Threshold Selection Method from Gray-Level Histogram. This method determines the threshold value by differentiating the two groups, ie objects and backgrounds, which have interlocking parts.

Otsu method is one method to segment (change the digital image of gray to black and white based on threshold value ratio with pixel color value of digital image) digital image. Threshold OTSU method of selecting the threshold value of the histogram (An image histogram is a graph depicting the deployment of pixel intensity values of a given image or part in a gray image).[8] Threshold value selection method is done by using statistical approach. The threshold value is selected from the gray color value located at the histogram interval where the gray color value maximizes the function value. The principle of Otsu method is described below. First, the probability of the intensity i in the histogram is calculated by:

$$
p(i)=\frac{n_{i}}{N}, p(i) \geq 0, \sum_{1}^{256} p(i)=1
$$

With $n_{i}$ denoting the number of pixels $i$ and $N$ intensity representing the number of all pixels in the image. If the histogram is divided into two classes (object and background), the weights in both classes are expressed as follows:

$$
\begin{aligned}
& w_{1}(t)=\sum_{i=1}^{t} p(i) \\
& w_{2}(t)=\sum_{i=t+1}^{L} p(i)=1-w_{1}(t)
\end{aligned}
$$

In this case, $L$ states the amount of the level of gray. The average of the two classes is calculated through:

$$
\begin{aligned}
& m_{1}(t)=\sum_{i=1}^{t} i \cdot p(i) / W_{1}(t) \\
& m_{2}(t)=\sum_{i=1}^{t} i \cdot p(i) / W_{2}(t)
\end{aligned}
$$

Calculate the optimum threshold value by maximizing ${\sigma_{B}}^{2}$ referred to as BCV (Between-Class Variance)

$$
\sigma_{B}{ }^{2}(t)=W_{1} \cdot\left[m_{1}(t)-m_{T}\right]^{2}+W_{2} \cdot\left[m_{2}(t)-m_{T}\right]^{2}
$$


In this case $m_{T}$ is the total mean

$$
\left(m_{T}=\sum_{i=1}^{N} i \cdot p(i)\right)
$$

The optimum threshold value can be obtained in two ways. The first way is implemented by minimizing Within-Class Variance. The second way is implemented by maximizing Between-Class Variance. However, in both ways, the second way is more computing.[9]

\section{Result and Discussion}

The data for this study used 30 (thirty) X-Ray image results from the data sets collected from several media sources, in the form of public data. X-Ray image data used taken from several data sources such as http://www.imageprocessingplace.com and also https://medpix.nlm.nih.gov. These data are then selected and collected in a Dataset folder which will be used as experiments using CLAHE algorithm combined with OTSU threshold.

\subsection{Image Quality Control Calculation}

This research uses Mean Square Error (MSE) which is a measure of quality control used to determine the quality of a digital image process to erorr value.[10] The MSE calculates how much data shifts between the source signal and the output signal, where the source and output signal have the same size. The value of a good MSE is close to 0 . The formula of the MSE calculation is:

$$
M S E=\sum_{i=1}^{n}(S-S e)^{2}
$$

Where $\mathrm{S}$ is the input signal $S, S e$ is the output signal, $\mathrm{n}$ is the length of the signal. The result of MSE calculation can be seen in graph of figure 1, with comparison between original CLAHE algorithm result and CLAHE algorithm with OTSU threshold combination.

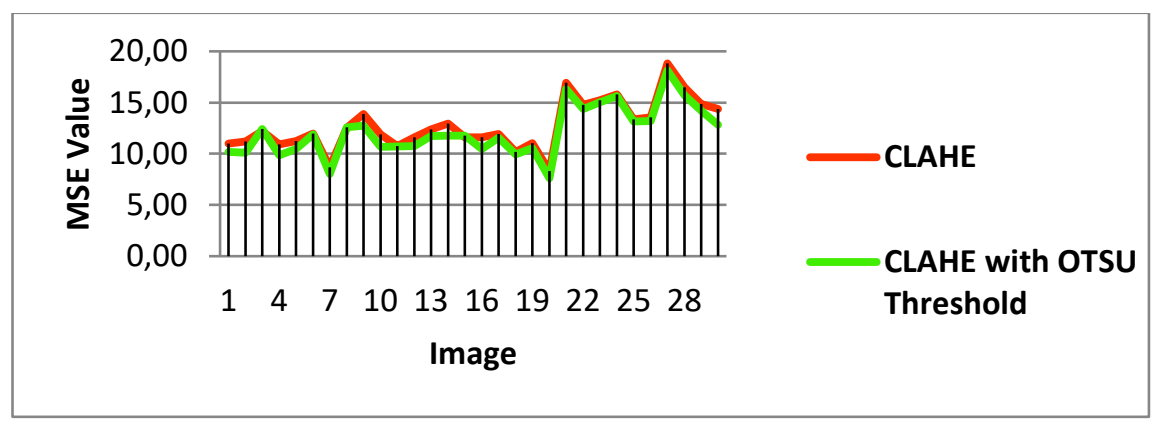

Fig 1. Comparison of MSE results between CLAHE and CLAHE blend with OTSU Threshold.

The results of the assessment using MSE from the comparison of two methods showed that the contribution made by the CLAHE algorithm combined with the OTSU Threshold 
produced a smaller error value than CLAHE, although the resulting error value was not too significant in image 3,8, and 15 .

\section{Conclusion}

Based on the results of research conducted by experimenting on CLAHE algorithm image filter whose cliplimit value is combined with OTSU Threshold which means no longer need the determination of parameter of limit value manually. The results of the error control measurement performance using MSE show the CLAHE algorithm combined with the contrast automation using OTSU Threshold the error value is reduced compared to the original CLAHE algorithm.

\section{References}

[1] S. Muniyappan, A. Allirani, and S. Saraswathi, "A novel approach for image enhancement by using contrast limited adaptive histogram equalization method," Comput. Commun. Netw. Technol. (ICCCNT),2013 Fourth Int. Conf., pp. 1-6, 2013.

[2] K. Koonsanit, S. Thongvigitmanee, N. Pongnapang, and P. Thajchayapong, "Image Enhanchement On Digital X-Ray Image Using N-CLAHE,” Biomed. Eng. Int. Conf., 2017.

[3] D. Mery, Computer vision for x-ray testing: Imaging, systems, image databases, and algorithms. 2015.

[4] M. Malarvel, G. Sethumadhavan, P. C. R. Bhagi, S. Kar, and S. Thangavel, "An improved version of Otsu's method for segmentation of weld defects on X-radiography images," Opt. - Int. J. Light Electron Opt., vol. 142, pp. 109-118, 2017.

[5] G. Jeon, "Color Image Enhancement by Histogram Equalization in Heterogeneous Color Space," Int. J. Multimed. Ubiquitous Eng., vol. 9, no. 7, pp. 309-318, 2014.

[6] A. A. Riadi, "Analisa Perbaikan Kualitas Kontras Citra X-Ray Menggunakan Metode Exposure Based Sub-Image Histogram Equalization,” SIMETRIS J. Tek. Mesin, Elektro dan Ilmu Komput., vol. 7, no. 1, pp. 305-310, 2016.

[7] J. Joseph, J. Sivaraman, R. Periyasamy, and V. R. Simi, "An objective method to identify optimum clip-limit and histogram specification of contrast limited adaptive histogram equalization for MR images," Biocybern. Biomed. Eng., vol. 37, no. 3, pp. 489-497, 2017.

[8] P. Wayalun, P. Chomphuwiset, N. Laopracha, and P. Wanchanthuek, "Images Enhancement of Gband Chromosome Using histogram equalization, OTSU thresholding, morphological dilation and flood fill techniques," Comput. Netw. Technol. (ICCNT), 2012 8th Int. Conf., pp. 163-168, 2012.

[9]S. Der Chen, "A new image quality measure for assessment of histogram equalization-based contrast enhancement techniques,” Digit. Signal Process. A Rev. J., vol. 22, no. 4, pp. 640-647, 2012.

[10] A. A. Riadi, A. A. Chamid, and A. Sokhibi, "Analisis komparasi metode perbaikan kontras berbasis histogram equalization pada citra medis," SIMETRIS J. Tek. Mesin, Elektro dan Ilmu Komput., vol. 8, no. 1, pp. 383-388, 2017. 